\title{
Verifying Object-Oriented Software: \\ Lessons and Challenges
}

\author{
K. Rustan M. Leino \\ Microsoft Research, Redmond, WA, USA \\ leino@microsoft.com
}

\begin{abstract}
A program verification system for modern software uses a host of technologies, like programming language semantics, formalization of good programming idioms, inference techniques, verification-condition generation, and theorem proving. In this talk, I will survey these techniques from the perspective of the Spec\# programming system, of which I will also give a demo. I will reflect on some lessons learned from building automatic program verifiers, as well as highlight a number of remaining challenges.
\end{abstract}

